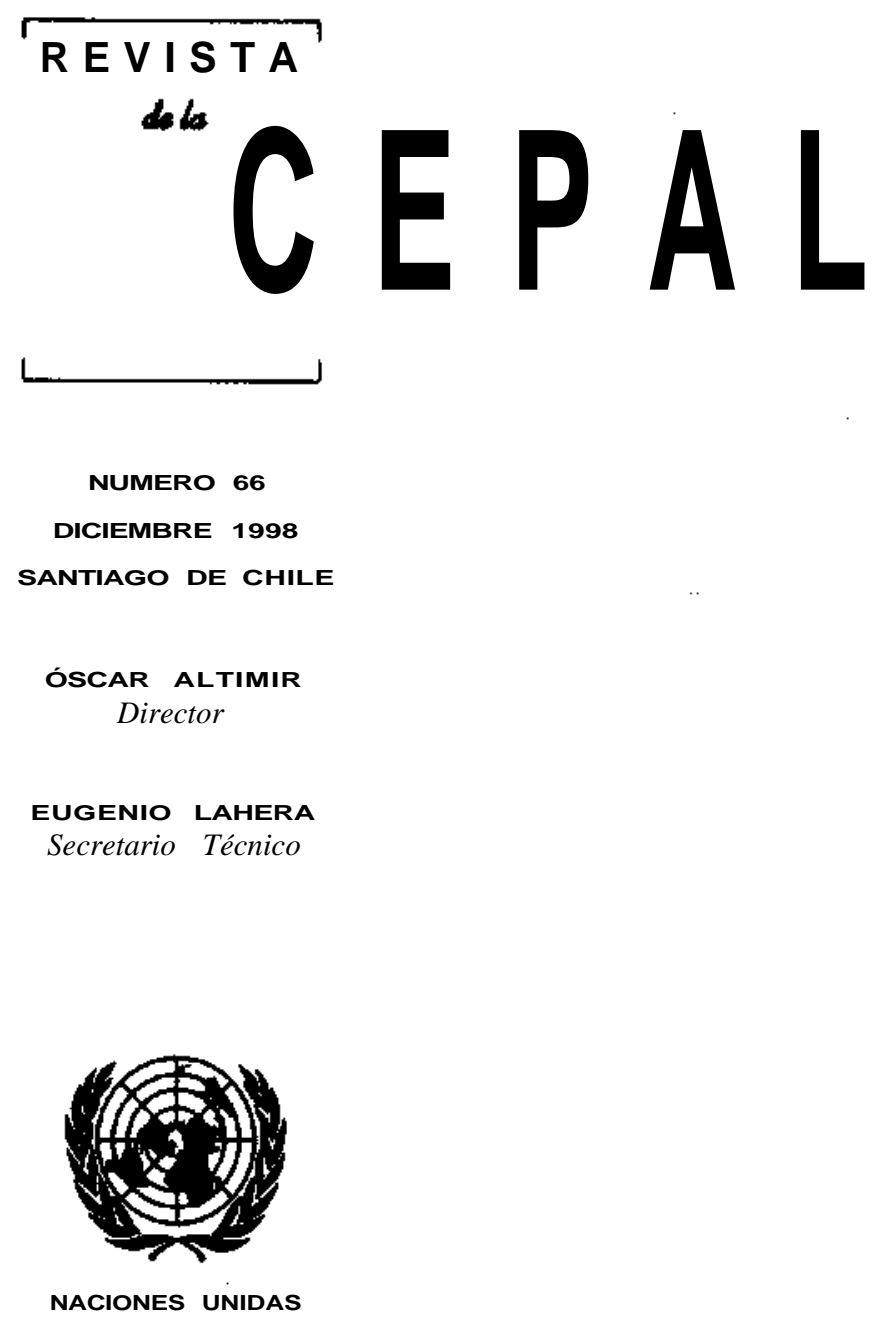


Más allá del Consenso de Washington: una visión desde la CEPAL

José Antonio Ocampo

La economía de Cuba

David ¡barra y Jorge Máttar

La educación en América Latina: la demanda y la distribución importan

Nancy Birdsall, Juan Luis Londoño y Lesley O'Connell

Determinantes de la desigualdad entre los hogares urbanos

Luis Felipe Jiménez L, y Nora Ruedi A.

Los compromisos de gestión en salud de Costa Rica con una perspectiva comparativa

Ana Sojo

Una estrategia de desarrollo a partir de complejos productivos en torno a los recursos naturales

Joseph Ramos

Grandes empresas y grupos industriales latinoamericanos

Celso Garrido y Wilson Peres

Entre el control político y la eficiencia: evolución de los derechos de propiedad agraria en México

Gustavo Gordillo, Alain de Janvry y Elizabeth Sadoulet

Los aranceles y el Plan Real de Brasil

Renato Baumann, Josefina Rivero y Yohana Zavattiero

Publicaciones recientes de la CEPAL 


\section{Determinantes de la \\ desigualdad entre los hogares urbanos}

Luis Felipe Jiménez $\mathrm{L}$. Nora Ruedi A.

Unidad de Financiamiento,

División de Comercio

Internacional, Transporte

y Financiamiento,

CEPAL
En este artículo se sintetizan los resultados de un análisis de factores de largo plazo que afectan a la distribución del ingreso en cinco países de la región: Argentina, Brasil, Chile, Colombia y México, sobre la base de encuestas de hogares disponibles para esos países entre 1979 y 1992. Tras una breve introducción (sección I), se examina la metodología aplicada (sección II) y luego se detallan los principales resultados (sección III). Se destacan entre éstos las menores remuneraciones al trabajo que perciben los primeros deciles de ingreso asociadas, entre otras cosas, a inequidades en los niveles educacionales alcanzados. A la vez, se observa que tales deciles exhiben tasas de ocupación inferiores al promedio y tasas de inactividad más altas. Este último fenómeno aparece vinculado a la composición de los hogares: los primeros deciles acusan una mayor presencia relativa de menores en el hogar, con lo cual la carga de cuidado de niños es mayor y los costos de participar en el mercado de trabajo se elevan, induciendo una menor participación en él y reduciendo la generación de ingresos. El artículo concluye con algunas sugerencias para una política redistributiva (sección IV). Esta debería avanzar conjuntamente en al menos cuatro esferas: generación de empleo productivo, elevación de los ingresos de los hogares más pobres, reducción de las barreras para que éstos accedan al mercado laboral y, finalmente, aspectos relacionados con la dinámica demográfica. 
I

\section{Introducción}

Tres son los motivos que originaron el presente artículo. En primer lugar, lograr mayores grados de equidad ha formado parte importante de los objetivos de la estrategia de desarrollo de los gobiernos de la región, que se expresa en propósitos de promover una mayor igualdad de oportunidades, así como en propuestas para reducir la incidencia de la pobreza. En segundo lugar, como ha sido documentado en otros estudios publicados por esta revista, el panorama distributivo a inicios de los años noventa, luego de los procesos de ajuste y reformas estructurales de los ochenta, exhibe un deterioro preocupante (Altimir, 1994) y su recuperación supone un importante desafío para los gobiernos y sus políticas. En tercer lugar, la evolución distributiva en la región durante Ja primera mitad de la década de los noventa continúa siendo desalentadora en términos generales, observándose cierto progreso en algunos casos aislados, CEPAL, (1994a y 1995). Aún más, el bajo crecimiento económico en ciertos casos, y el consiguiente incremento en las tasas de desocupación (OIT, 1996), hacen prever que el deterioro distributivo podría agravarse en determinados países.

En virtud de lo anterior, cobran renovada importancia políticas orientadas a superar esta situación, tomando en cuenta tanto el carácter de largo plazo de ciertos factores que afectan la distribución del ingreso, ${ }^{1}$ como la urgencia de adoptar medidas que en el mediano plazo permitan mejorar la situación de los grupos más pobres, por la gravedad de su condición.

D Este documento resume resultados de investigaciones realizadas en el marco del proyecto Gobierno de Holanda/CEPAL sobre "Distribución del ingreso y pobreza en experiencias recientes de estabilización y ajuste en países de América Latina y el Caribe". Los análisis individuales para Argentina, Brasil, Chile, Colombia y México fueron publicados en forma separada (Jiménez y Ruedi, 1997 a,b,c,d y e). Para la elaboración de estos estudios se contó con el apoyo de la División de Estadística y Proyecciones Económicas de la CEPAL, Los autores agradecen los comentarios de Oscar Altimir, Juan Carlos Feres y Carlos Howes, así como la colaboración que ellos brindaron.

Respecto de la influencia sobre la distribución del ingreso de factores y políticas de corto plazo, véase Jiménez (1997).
Este artículo aborda una de las dimensiones de la equidad, analizando factores que contribuyen a dar cuenta de las diferencias en el ingreso per cápita de los hogares en cinco países de la región. Diversos estudios de la distribución del ingreso se han orientado a examinar factores que explicarían las desigualdades entre perceptores de ingreso. En contraste, en el presente estudio la unidad de análisis son los hogares, que incluyen tanto a personas que perciben ingresos así como a otras que no lo hacen. El análisis se centra entonces en determinar cómo se conforma el ingreso per cápita del hogar. Las variables consideradas incluyen los niveles de remuneración, la incorporación al empleo y las tasas de participación de los miembros del hogar, así como también aspectos demográficos que influyen sobre el tamaño de los hogares. También se examinan aspectos relacionados con desigualdades en las remuneraciones de los factores, asociadas a características de los perceptores, pero principalmente en su relación con el ingreso per cápita del hogar.

Debe tenerse presente que, aun cuando se emplea una metodología común, el análisis no se orienta a establecer comparaciones distributivas entre países; el propósito es más bien identificar regularidades en el comportamiento de ciertas variables que contribuyen a explicar la distribución del ingreso en un determinado país. A la vez, con el fin de obtener conclusiones de cierta validez general, esta síntesis se apoya en resultados obtenidos para los principales centros urbanos de los cinco países. Como la cobertura de las encuestas varía mucho de un país a otro, ${ }^{2}$ la opción de restringir el análisis a estos centros aparece como válida al momento de comparar resultados con el fin de establecer conclusiones aplicables a otras experiencias.

\footnotetext{
2 A modo de ejemplo, los resultados para Argentina se refieren a Buenos Aires, en tanto que las encuestas en Brasil y Chile incluyen zonas rurales además de las metropolitanas.
} 


\section{II \\ El método de análisis}

Con miras a reforzar el carácter comparativo de este estudio, se empleó una metodología anteriormente utilizada por Pollack y Uthoff (1990) en el examen de la distribución del ingreso en el Gran Santiago, Chile, En el presente caso la metodología se aplicó a encuestas de hogares disponibles entre 1979 y 1992 en Argentina, Brasil, Chile, Colombia y México. Esta parte de una identidad sencilla, pero que posee el mérito de identificar algunos factores claves que afectan el nivel de ingreso del hogar. La metodología es básicamente descriptiva y busca detectar regularidades de comportamiento de determinadas variables asociadas a la distribución del ingreso. No se trata en consecuencia de la aplicación de un enfoque teórico que presupone $a$ priori cierto orden de causalidad, aunque la interpretación de los resultados, basada en la identificación de diferencias sistemáticas de comportamiento entre deciles, se apoya en hipótesis explicativas de los orígenes de la desigualdad y de sus cambios.

El ingreso per cápita del hogar puede ser expresado de manera simplificada por la siguiente identidad:

$Y p c=\frac{Y T}{N}=\frac{Y P}{L} \times \frac{L}{L+D} \times \frac{L+D}{P E T} \times \frac{P E T}{N} \times \frac{O Y}{N}$

donde:

$Y p c$ = ingreso per cápita del hogar;

$\mathrm{YT}=$ ingreso total disponible del hogar;

$N=$ número de personas en el hogar;

$Y P=$ ingreso primario de los ocupados del hogar;

$L \quad$ = número de personas ocupadas del hogar;

$D$ = número de personas desempleadas del hogar;

$P E T$ - número de personas del hogar en edad de trabajar;

$O Y$ - otros ingresos del hogar.

De este modo, el ingreso per cápita del hogar queda expresado en función de variables económicas y demográficas. El primer término del lado derecho, $Y P / L$, corresponde a la remuneración media obtenida por las personas ocupadas del hogar. Este ingreso primario se compone a su vez de ingresos del trabajo (sueldos, salarios e ingresos del trabajo por cuenta propia). Corresponde entonces a un concepto de remuneración factorial media por ocupado.
El segundo término del lado derecho, $L /(L+D)$, es la tasa de ocupación del hogar, siendo su complemento la tasa de desocupación. El tercer término, $(L+D)$ / $P E T$, es la tasa de participación. El cuarto término es la razón entre el número de personas del hogar en edad de trabajar y el tamaño del hogar, PET/N. El inverso de este factor es una aproximación a la tasa de dependencia del hogar, en el sentido que se discute en el párrafo siguiente. Mientras mayor sea esta razón, mayor será el número de personas en edad de dar sustento económico al hogar y con ello menor será la tasa de dependencia.

Este concepto de tasa de dependencia tiene una connotación demográfica, en contraste con otros usos que destacan aspectos diferentes. Así, es frecuente referirse a esta tasa como la razón entre los ocupados y el número de personas que no trabajan en cierto grupo, haciendo con ello hincapié en el esfuerzo económico que efectivamente deben hacer los ocupados para dar sustento al resto. Las variables relevantes en dicho caso serán las tasas de ocupación y de salarios, las que ya se incluyen en los dos primeros factores de la ecuación. En el presente caso, la razón entre las personas en edad de trabajar y el tamaño del hogar, representa la proporción del hogar que potencialmente podría sustentarlo, siendo la estructura de edades y el tamaño del hogar las variables relevantes.

Finalmente, el último factor corresponde a ingresos percibidos de otras fuentes, distintas al trabajo, es decir, ingresos por concepto de propiedad y transferencias, ambos en términos per cápita. Las transferencias corresponden a subsidios recibidos e ingresos por jubilaciones.

Las diferencias por deciles en los niveles de ingreso per cápita de las familias quedan entonces expresadas en función de los comportamientos que exhiben las tasas de remuneración factorial, de ocupación y de participación en la fuerza de trabajo, una variable relativa a la estructura demográfica del hogar y un componente de otros ingresos. Los cambios en la distribución del ingreso se asocian así a la evolución de estos componentes a través del tiempo. En consecuencia, los hogares se ordenaron según su ingreso per cápita y se calcularon los factores componentes de esta identidad. No obstante, dado que estos últimos resumen la influen- 
cia de muchas variables, se obtuvieron indicadores adicionales que contribuyeran a explicar las diferencias en los niveles de ingreso primario, tasas de participación y estructura de los hogares.

Para una mejor comprensión de las diferencias según deciles en los ingresos primarios, siempre con los hogares ordenados de acuerdo con el ingreso per cápita, se calcularon sus componentes, es decir, sueldos y salarios medios de los asalariados del hogar y los ingresos medios de los trabajadores independientes. También se obtuvieron los ingresos medios provenientes de la propiedad, completando de este modo las fuentes factoriales de ingreso.

Otros enfoques, teóricos y aplicados, se han orientado a explicar las diferencias en los ingresos entre perceptores en función de características relacionadas con el nivel educacional de los oferentes de trabajo. En este estudio, con el fin de explorar la contribución del mencionado factor a la explicación de la desigualdad de ingreso per cápita entre deciles de hogares, se procedió a examinar la estructura de los hogares de cada decil según la educación del jefe de hogar.

También, con el fin de aproximarse a variables que causan diferencias en las tasas de ocupación y participación, se examinó el comportamiento de los hogares en cada decil según la actividad del jefe de hogar y del cónyuge. En este último caso, los cálculos registrados se restringen a cónyuges de jefes de hogar ocupados, con el fin de examinar la capacidad de ambos para otorgar sustento económico al hogar.

Finalmente, con el propósito de indagar acerca de la estructura de los hogares y su posible influencia sobre otras variables, se obtuvo, por deciles de hogares, la proporción de niños con relación a los adultos y el número medio de personas en el hogar.

Es pertinente realizar ciertos alcances relativos al concepto de ingreso que aquí se emplea. En primer lugar, las cifras utilizadas son aquellas que recogen los ajustes efectuados por la CEPAL, que se orientan a compatibilizar esta información con aquella proveniente de las cuentas nacionales. En términos generales el procedimiento consiste en realizar imputaciones de ingreso con el fin de corregir discrepancias que surgen al comparar las partidas de la cuenta de hogares del sistema de cuentas nacionales, con aquellas obtenidas de la encuesta (Feres, 1995; CEPAL, 1991 y 1996b).

En segundo lugar, las encuestas registran el ingreso una vez descontados los impuestos y las contribuciones a la seguridad social, pero incluyen transferencias y donaciones recibidas. En tercer lugar, se ha excluido el alquiler imputado, ya que la imputación se realiza a nivel de los hogares en tanto que los indicadores seleccionados se refieren principalmente a características medias de los miembros del hogar, referentes a su capacidad de generación de ingreso.

Por último, el ingreso captado por las encuestas incluye aquel atribuible a la propiedad. No obstante, la experiencia señala que éstas reflejan los ingresos provenientes de la propiedad en una proporción considerablemente menor que en el caso de los otros componentes del ingreso. Como las dificultades de medición de esta índole son agravadas por cuadros inflacionarios importantes, como los que afectaron a países de la región durante el período examinado, es arriesgado generalizar sobre la base de estos datos. En consecuencia, el análisis se centra en torno a los otros componentes del ingreso del hogar.

Dado el interés que suscita la distribución del ingreso y los factores explicativos, los cuadros estadísticos presentan índices relativos, empleando el promedio de cada variable en el total de la muestra como patrón de referencia. Esto permite ilustrar el grado en que la variable media en cada decil difiere respecto del conjunto de la muestra.

\section{III}

\section{Principales resultados comparativos}

En el cuadro] se presentan sucintamente algunos rasgos relativos a la distribución del ingreso entre 1980 y 1992 en los principales centros urbanos ${ }^{3}$ de los cinco países estudiados. Como allí se aprecia, durante este

\footnotetext{
? En el caso de México se trata de zonas de alta densidad.
}

período, que en su mayor parte se caracterizó por importantes fluctuaciones macroeconómicas asociadas a la crisis de endeudamiento externo que afectó a países de la región, los cambios en la distribución del ingreso fueron significativos. De acuerdo al coeficiente de Gini, la concentración del ingreso en los principales centros urbanos se redujo en Colombia, aumentó en 
América Latina (cinco países): Indicadores de la distribución del ingreso

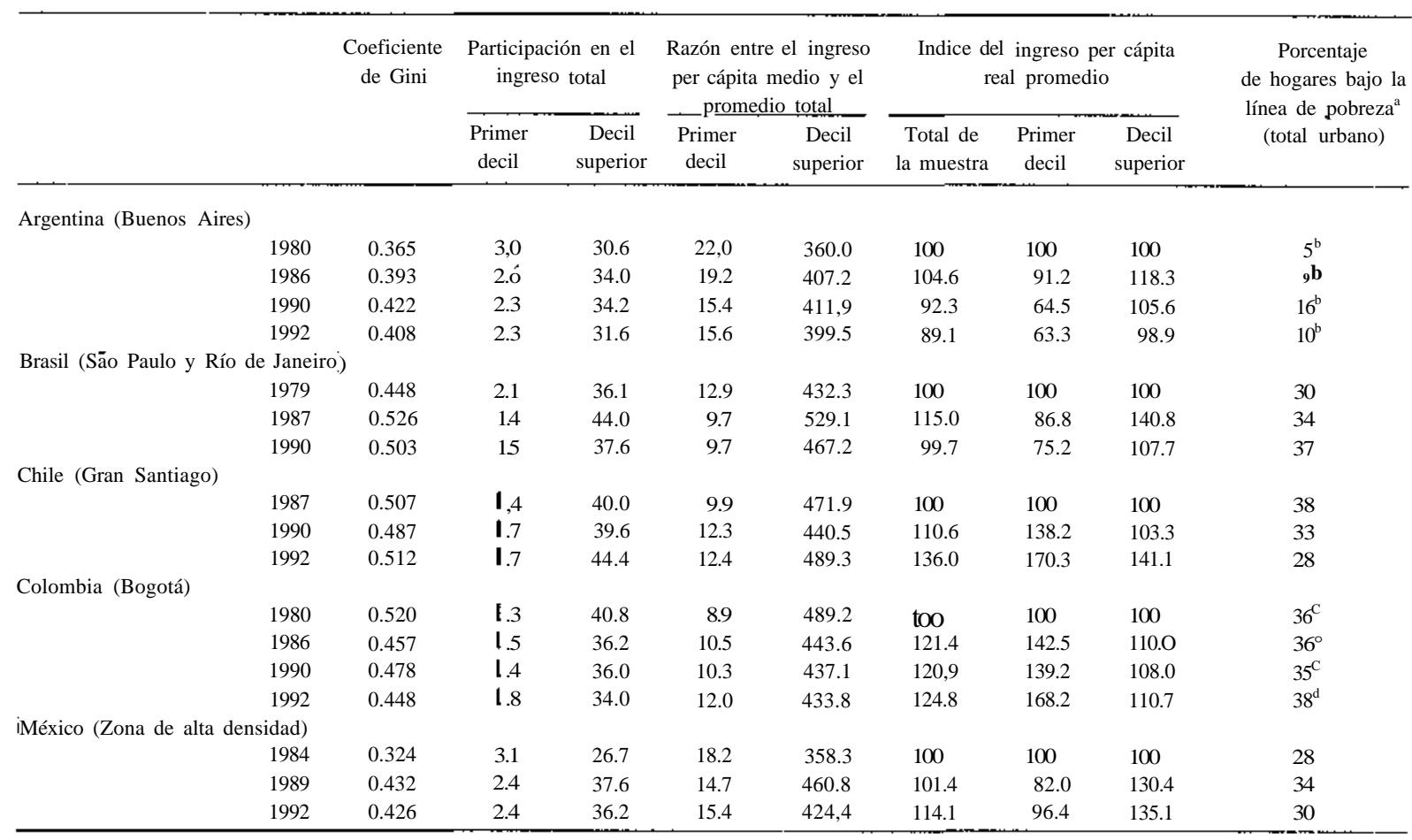

Fuente: CEPAL sobre la base de tabulados especiales de encuestas de hogares.

${ }^{a}$ CEPAL 1997. ${ }^{b}$ Áreas metropolitanas. ${ }^{c}$ Siete ciudades principales, ${ }^{d}$ Ocho ciudades principales.

Argentina, Brasil y México y tendió a mantenerse con ciertas oscilaciones en Chile.

Más ilustrativo resulta observar los cambios en las participaciones en el ingreso total de los deciles que ocupan ambos extremos de la distribución. ${ }^{4}$ Examinando el caso de Colombia, país que exhibe reducciones en la concentración durante este período, se aprecia una redistribución de alrededor de $7 \%$ del ingreso desde el decil superior principalmente hacia deciles medios altos, elevándose la participación de los deciles inferiores en una proporción reducida (véase Jiménez y Ruedi, 1997b). Debe hacerse notar, sin embargo, que redistribuciones de esta magnitud son muy significativas; aplicadas a un PIB en crecimiento, representan una importante transferencia de ingresos hacia sectores medios altos. Otros indicadores reflejan una evolución

\footnotetext{
4 Solamente con fines ilustrativos, diremos que la mayoría de los cuadros incluidos en el texto presentan información relativa a los deciles extremos, pese a que las conclusiones se basan en la observación de toda la distribución, tal como aparece en los estudios de casos nacionales que este artículo sintetiza.
}

un tanto más positiva. Así, el ingreso per cápita medio del primer decil pasó de representar un $8.9 \%$ del promedio total a un $12 \%$. A su vez, el ingreso real de este segmento se expandió en $68.2 \%$, mientras que el total subió $24.8 \%$. No obstante, probablemente debido al bajo nivel inicial del ingreso per cápita de los estratos inferiores, el aumento del ingreso no fue suficiente para reducir la incidencia de la pobreza en los hogares urbanos. En síntesis, en Colombia se verificó un proceso redistributivo, pero que no alcanzó significativamente a los estratos pobres. ${ }^{5}$

En los países en que aumentó la concentración en este período (Argentina, Brasil y México) se observan reducciones en la participación del decil inferior y, en ciertos casos, significativas redistribuciones en favor del decil superior, incrementos en las diferencias de ingreso per cápita entre el decil inferior y superior respecto del promedio, pérdidas de ingreso real en el pri-

\footnotetext{
5 Ocampo, Pérez, Tovar y Lasso (1998) señalan que si bien la distribución del ingreso mejoró en los sectores urbanos, en los rurales se apreció un deterioro.
} 
mer decil y aumentos en la incidencia de la pobreza.

A su vez, en Chile se observan variaciones distributivas que no se expresan totalmente en cambios del coeficiente de Gini. En efecto, la participación del decil inferior se incrementa levemente pero en menor grado que los aumentos de participación del decil superior. También, si bien como porcentaje del promedio se eleva el ingreso per cápita del primer decil, aquel del estrato más alto también sube, distanciándose así aún más del resto. En el contexto del crecimiento experimentado, el ingreso real per cápita del primer estrato se expande a una tasa superior al promedio y concomítantemente se aprecia una reducción del porcentaje de hogares en situación de pobreza. Sin embargo, el decil más alto también exhibe mejoras superiores al promedio, contrarrestando de este modo una posible mejoría en la distribución. En suma, como otros análisis han señalado (CEPAL, 1997), la experiencia chilena podría ser considerada como un proceso de crecimiento con reducción de la pobreza y bajo contenido redistributivo. Más adelante se establecerán hipótesis que contribuirían a explicar el bajo grado de difusión social del crecimiento que se ha observado en algunas experiencias de la región.

En suma, con la excepción de Colombia, se aprecia un incremento en las diferencias de ingreso entre ambos extremos de la distribución durante el período examinado. No obstante, en todos los casos la participación de los primeros deciles permanece en niveles muy bajos. Nuevamente con la salvedad de Colombia, las redistribuciones de ingreso se tradujeron en incrementos de la participación del decil superior que resultan muy significativas, equivalentes en algunos casos a entre dos y tres veces la participación total del decil inferior. Además, se destaca la gran persistencia en el tiempo de las bajas participaciones en el ingreso de los grupos más pobres, fenómeno que se repite en todos los países, otorgándole una gran inercia a la distribución. Finalmente, durante el período examinado sólo se observa reducción de la pobreza urbana en Chile, en tanto que en los países restantes se incrementa su incidencia o bien la situación se estanca.

\section{Rasgos estilizados de los niveles relativos de ingreso}

\section{a) Componentes del ingreso primario}

En el cuadro 2 se presentan antecedentes relativos a los componentes del ingreso primario y las transferencias. Se confirma allí la influencia sobre la distribución de las remuneraciones al trabajo asalariado.
En efecto, en aquellos países en los cuales son menores las diferencias relativas en esta variable (Argentina y México), tiende a ser mayor la participación del primer decil en el ingreso. A su vez, desde una perspectiva temporal, en los países en que se deterioró la distribución (Argentina, Brasil y México), se profundizaron las diferencias relativas de las remuneraciones salariales por ocupado.

El ingreso medio del trabajo independiente exhibe diferencias relativas entre deciles aún más pronunciadas que en el caso anterior y han tendido generalmente a profundizarse en el tiempo (cuadro 2). Típicamente, estos ingresos representan una fracción creciente del total por deciles, lo que se debería a la mayor heterogeneidad del tipo de trabajo que da origen a esta fuente de ingreso. Así, en esta categoría de ingresos suelen incluirse, entre los estratos superiores, aquellos provenientes del empleo profesional independiente y de rentas de empleadores, en tanto que entre los estratos inferiores éstos son generados mayormente por trabajadores por cuenta propia de baja calificación.

En suma, la desigualdad del ingreso per cápita de los hogares corresponde en gran medida a disparidades en las remuneraciones del trabajo, aunque este no es el único factor determinante, como más adelante se examina. A su vez, cambios en las diferencias en las remuneraciones relativas se expresan en fluctuaciones distributivas, asociándose una reducción de éstas a una mayor participación en el ingreso de los deciles más pobres.

Los factores que influyen en las diferencias de remuneraciones son de variada índole y han sido abordados por diversos estudios. En primer lugar, el resultado que con mayor solidez se obtiene se refiere a la influencia de la variable educacional. ${ }^{6}$ El cuadro 3 presenta la distribución del ingreso en Brasil, Chile y Colombia según el nivel educacional de los jefes de hogar, por deciles. ${ }^{7}$ Como allí se aprecia, existe una asociación sistemática entre los niveles de educación alcanzados y el decil de ingreso al cual pertenece el jefe del hogar; mientras más alto sea su nivel educacional, más probabilidades tendrá el hogar que encabeza de pertenecer a un decil cuyo ingreso per cápita sea mayor.

\footnotetext{
6 Véase por ejemplo Altimir y Piñera (1977); también Psacharopoulos, Morley, Fiszbein, Lee y Wood (1992).

La información para Argentina y México se presenta clasificada según niveles educacionales distintos a los presentados en este cuadro, por lo que no son comparables. No obstante, los resultados se mantienen. Véanse los estudios de caso para dichos países en esta misma serie.
} 
CUADRO 2

América Latina (cinco países): índices relativos de factores componentes del ingreso del hogar ${ }^{8}$

\begin{tabular}{|c|c|c|c|c|c|c|c|c|}
\hline & \multicolumn{2}{|c|}{$\begin{array}{c}\text { Ingreso primario } \\
\text { medio/ingreso } \\
\text { medio total }\end{array}$} & \multicolumn{2}{|c|}{$\begin{array}{c}\text { Ingreso asalariado } \\
\text { medio/ingreso } \\
\text { medio total }\end{array}$} & \multicolumn{2}{|c|}{$\begin{array}{l}\text { Ingreso medio del } \\
\text { trabajo independíenle/ } \\
\text { ingreso medio total }\end{array}$} & \multicolumn{2}{|c|}{$\begin{array}{l}\text { Ingreso rnedio por } \\
\text { transferencias/ingreso } \\
\text { medií) total }\end{array}$} \\
\hline & $\begin{array}{c}\text { Primer } \\
\text { decil }\end{array}$ & $\begin{array}{c}\text { Decil } \\
\text { superior }\end{array}$ & $\begin{array}{c}\text { Primer } \\
\text { decil }\end{array}$ & $\begin{array}{c}\text { Decil } \\
\text { superior }\end{array}$ & $\begin{array}{c}\text { Primer } \\
\text { decil }\end{array}$ & $\begin{array}{c}\text { Decil } \\
\text { superior }\end{array}$ & $\begin{array}{c}\text { Primer } \\
\text { decil }\end{array}$ & $\begin{array}{c}\text { Decil } \\
\text { superior }\end{array}$ \\
\hline \multicolumn{9}{|l|}{ Argentina (Buenos Aires) } \\
\hline 1980 & 40.1 & 241.3 & 60.9 & 202.5 & 9.4 & 350.8 & 61.7 & 172.2 \\
\hline 1986 & 32.0 & 303.2 & 38.6 & 199.0 & 14.2 & 467.0 & 87.1 & 153.7 \\
\hline 1990 & 25.9 & 321.8 & 39.8 & 202.0 & 6.8 & 452.3 & 86.9 & 119.9 \\
\hline 1992 & 25.3 & 291.4 & 41.0 & 181.2 & 4.1 & 397.4 & 83.3 & 185.4 \\
\hline \multicolumn{9}{|l|}{ Brasil (São Paulo y Río de Janeiro) } \\
\hline 1979 & 26.9 & 323.1 & 32.1 & 289.9 & 9.0 & 471.3 & 21.3 & 362.4 \\
\hline 1987 & 14.8 & 385.7 & 23.6 & 323.4 & 7.5 & 478.3 & 20.2 & $462.9^{\circ}$ \\
\hline 1990 & 19.9 & 342.9 & 22.7 & 305.5 & 10.1 & 452.3 & 19.3 & 435.2 \\
\hline \multicolumn{9}{|l|}{ Chile (Gran Santiago) } \\
\hline 1987 & 16.4 & 422.4 & 21.0 & 325.5 & 9.3 & 655.3 & 22.8 & 287.6 \\
\hline 1990 & 23.4 & 401.3 & 28.5 & 321.5 & 9.4 & 551.3 & 30.4 & 224.5 \\
\hline 1992 & 23.2 & 448.9 & 32.8 & 320.1 & 7.0 & 602.3 & 21.3 & 301.7 \\
\hline \multicolumn{9}{|l|}{ Colombia (Bogotá) } \\
\hline 1980 & 18.1 & 391.6 & 24.1 & 302.9 & 9.1 & 465.3 & 42.2 & 315.1 \\
\hline 1986 & 22.6 & 327.1 & 29.1 & 271.4 & 8.7 & 421.6 & 17.0 & 374.0 \\
\hline$! 990$ & 23.0 & 317.7 & 25.5 & 239.5 & 9.6 & 384.5 & 10.4 & 392.9 \\
\hline 1992 & 31.1 & 308.0 & 37.3 & 254.5 & 14.6 & 371.7 & 6.7 & 398.8 \\
\hline \multicolumn{9}{|l|}{ México (Zona de alta densidad) } \\
\hline 1984 & 35.4 & 262.3 & 42.7 & 188.2 & 16.3 & 372.4 & 42.0 & 259.1 \\
\hline 1989 & 29.8 & 377.5 & 45.3 & 210.8 & 7.5 & 573.7 & 23.0 & 318.5 \\
\hline 1992 & 27.6 & 411.3 & 41.6 & 243.9 & 7.7 & 614.7 & 25.9 & 242.6 \\
\hline
\end{tabular}

Fuente: CEPAL, sobre la base de tabulados especiales de encuestas de hogares.

${ }^{a}$ Los índices relativos son razones entre el valor medio de la variable en determinado decil y el correspondiente promedio.

CUADRO 3

América Latina (tres países): Distribución de los hogares por años de estudios del jefe de hogar, por deciles

(Porcentajes)

\begin{tabular}{|c|c|c|c|c|c|c|c|c|c|c|c|c|}
\hline & \multicolumn{4}{|c|}{ Brasil, $1990^{\mathrm{a}}$} & \multicolumn{4}{|c|}{ Colombia $1992^{\mathrm{b}}$} & \multicolumn{4}{|c|}{ Chile, $1992^{\mathrm{a}}$} \\
\hline & 0 a3 años & 4 a 9 años & 10 a 12 años & 13 y más & 0 a 3 años & 4 a 9 años & 10 a 12 años & 13 y más & 0 a 3 años & 4 a 9 años & 10 a 12 años & 13 y mas años \\
\hline Tota! & 100 & 100 & 100 & 100 & 100 & 100 & 100 & 100 & 100 & 100 & 100 & 100 \\
\hline 1 & 17.9 & 5.3 & 1.2 & 0.1 & 18.3 & 12.7 & 4.7 & 1,0 & 14.5 & 13.3 & 6.8 & 2.1 \\
\hline 2 & 16.5 & 6.7 & 1.7 & 0.3 & 15.6 & 12.7 & 6.5 & 1.1 & 12.5 & 12.5 & 9.0 & 2.2 \\
\hline 3 & 14.2 & 9.0 & 3.0 & 0.4 & 15.2 & 12.3 & 7.2 & 1.7 & 12.5 & 11.6 & 9.9 & 3.1 \\
\hline 4 & 12.7 & 10.3 & 4.4 & 0.6 & 14.5 & 12.5 & 6.6 & 2.0 & 14.1 & 11.1 & 9.6 & 3.4 \\
\hline 5 & 11.3 & 11.4 & 6.1 & 0.9 & 14,0 & 11.6 & 8.7 & 3.4 & 12.5 & 10.9 & 9.5 & 5.9 \\
\hline 6 & 9.5 & 12.8 & 7.8 & 1.9 & 8.8 & 11.9 & 11.0 & 5.0 & 11.7 & 10.7 & 10.3 & 5.6 \\
\hline 7 & 7,8 & 13.2 & 11.3 & 3.9 & 6.2 & 10.0 & 12.8 & 9.5 & 8.5 & 10.1 & 11.2 & 9.2 \\
\hline 8 & 5.4 & 13.5 & 18.2 & 8.2 & 3.8 & 8.1 & 15.7 & 13.9 & 7.0 & 8.9 & 12.0 & 12.5 \\
\hline 9 & 3.2 & 11.5 & 23.2 & 23.2 & 2.7 & 5.4 & 15.2 & 24.3 & 4.9 & 7.3 & 11.8 & 19.7 \\
\hline 10 & 1.4 & 6.4 & 23.0 & 60.5 & 0.8 & 2.9 & 11.7 & 38.2 & 1.8 & 3.8 & 9.9 & 36.3 \\
\hline
\end{tabular}

Fuente: CEPAL, sobre la base de tabulados especiales de encuestas de hogares.

${ }^{a}$ Total nacional. a Ocho ciudades principales. 
En segundo lugar, en el contexto de mercados segmentados, la remuneración al capital humano también difiere según el segmento del mercado de trabajo en que se inserte el trabajador. Así, para un mismo nivel de calificación, la remuneración dependerá del tipo de ocupación, el tamaño y la propiedad de la empresa (Altimir y Piñera, 1977). Existe además suficiente información que señala que los grupos de menores ingresos se insertan en mayor grado en sectores de baja productividad y crecimiento, originando menores remuneraciones para un mismo nivel de calificación. Así, entre el 40 y el $60 \%$ de la población urbana ocupada está adscrita a segmentos de baja productividad, principalmente la microempresa y el trabajo independiente no calificado (CEPAL, 1996a).

En síntesis, las diferencias en el ingreso per cápita de los hogares resultan parcialmente de desigualdades en la remuneración al trabajo, originadas a su vez por diferencias en los niveles de calificación y adscripción a los segmentos menos dinámicos del mercado de trabajo.

\section{b) El nivel relativo de transferencias}

En el cuadro 2 aparece también información sobre transferencias medias por perceptor (jubilaciones, pensiones y otros ingresos) en los deciles extremos, a la vez que se documenta su evolución relativa en el período analizado. Resaltan al respecto los resultados de Argentina, que exhibe menores diferencias relativas; aún más, en el tiempo dichas transferencias habrían contribuido a evitar en este caso un mayor deterioro distributivo, por cuanto tendieron a acortarse las distancias entre los deciles inferior y superior. Aun así, se observa el mismo comportamiento sistemático que en otros países; las transferencias medias por perceptor del hogar tienden a elevarse con el nivel de ingreso, estableciéndose diferencias que en algunos casos son muy significativas. Estas desigualdades se explicarían por la interacción de varios factores. En primer lugar, la cobertura de los sistemas de previsión social generalmente es menor entre segmentos de menores ingresos. En segundo lugar, las jubilaciones o pensiones otorgadas tienden a reflejar la desigualdad de ingresos prevaleciente al momento de adquirirse el derecho a ellas. En tercer lugar, las pensiones asistenciales, al ser de cargo público, frecuentemente han sido erosionadas por procesos inflacionarios. Finalmente, la mayor informalidad entre los grupos de menores ingresos dificulta su cobertura por parte de los organismos públicos de protección y asistencia social. Vemos así que, por lo general, las transferencias tienden a ser insuficientes para compensar las inequidades de la distribución primaria.

\section{Tasas de ocupación, participación y estructu- ra del hogar}

La influencia de las disparidades en las tasas de ocupación sobre la distribución del ingreso queda de manifiesto tanto en los análisis de corte transversal como en una perspectiva dinámica. Como se ilustra en el cuadro 4, los primeros estratos de ingreso exhiben tasas de ocupación consistentemente menores que el promedio y éstas son crecientes a medida que se sube en la escala de ingresos. A su vez, allí donde se observó un aumento de la concentración del ingreso (Argentina, Brasil y México), los niveles relativos de ocupación del primer decil se deterioraron, en tanto que aquellos del decil superior mejoraron. En contraste, en el caso de Colombia (Bogotá), la mejoría distributiva se presenta asociada a un aumento en el nivel relativo de ocupación de los primeros estratos, reduciéndose así la distancia respecto del estrato superior.

Un comportamiento similar al anterior exhiben las tasas de participación, con la salvedad de que, para cada año, su dispersión es mayor que la de las correspondientes tasas de ocupación. Como muestra el cuadro 4, las tasas de participación son persistentes y sustancialmente inferiores al promedio entre los hogares más pobres. Por otra parte, sólo en Colombia (Bogotá) se aprecian reducciones en el tiempo de las disparidades de tasas de participación, siendo este el único caso en que disminuye la concentración del ingreso. Allí donde aumentó la concentración (Argentina, Brasil y México) persisten las diferencias en esta variable. En Chile, país que no presenta mejoras distributivas no obstante su crecimiento sostenido y los aumentos en las tasas de ocupación, se incrementó en términos relativos la desigualdad en las tasas de participación. ${ }^{8}$ En efecto, no obstante un incremento a nivel absoluto, en términos relativos la tasa de participación del primer decil se redujo respecto del promedio debido a una mayor alza en la participación de los estratos medios altos. De este modo, el crecimiento no se tradujo en la misma medida en ganancias distributivas.

El párrafo precedente se refiere a la participación en el mercado de trabajo de los integrantes del hogar

\footnotetext{
${ }^{8}$ Para un mayor detalle de la evolución de esta variable en Chile, véase el correspondiente estudio de caso.
} 
América Latina (cinco países): índices relativos* de factores que reflejan la participación en el mercado $<$ Je trabajo

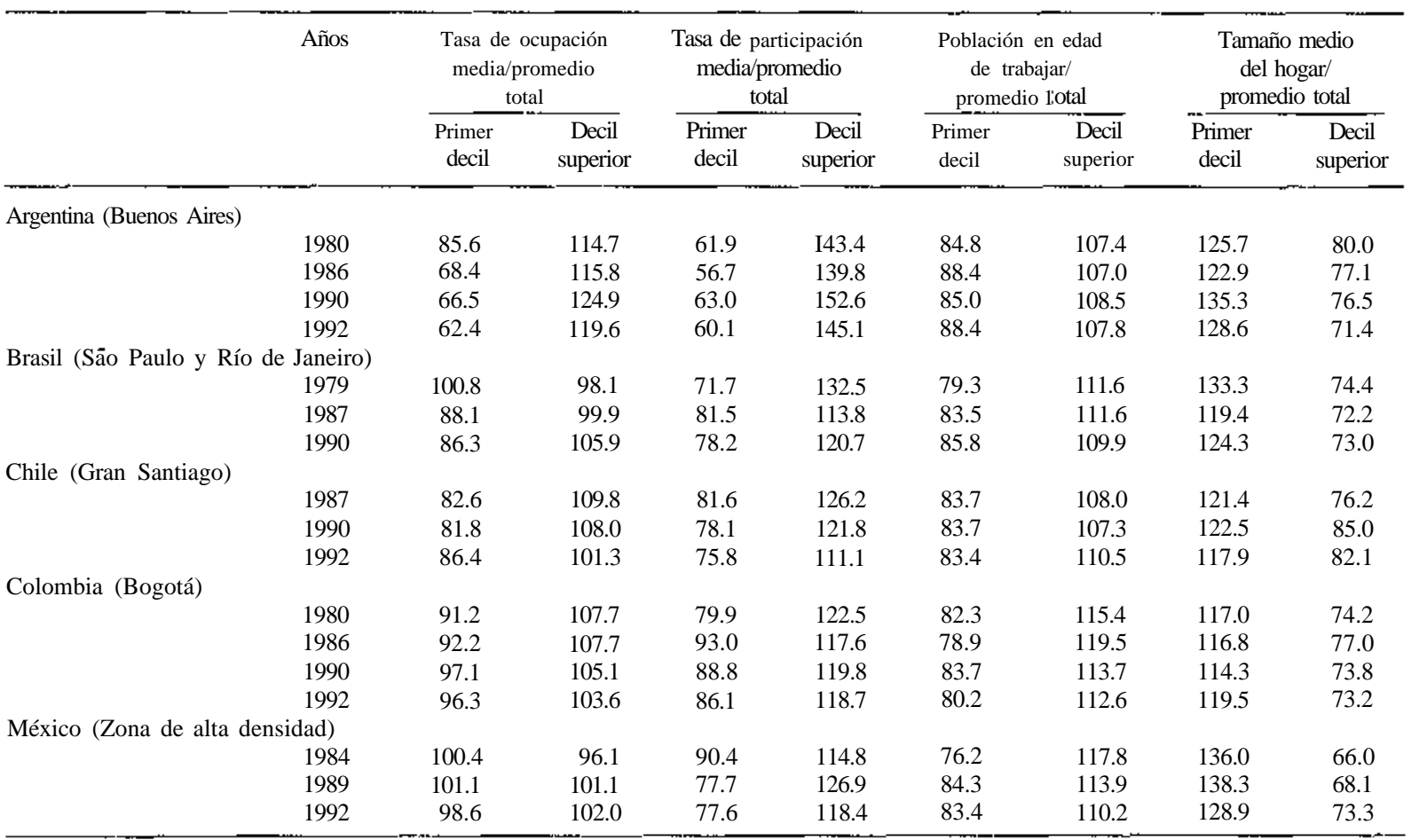

Fuente.- CEPAL, sobre la base de tabulados especiales de encuestas de hogares.

a Razones entre el valor medio de la variable en determinado decil y el correspondiente promedio.

pertenecientes a la población en edad de trabajar. Sin embargo, el tamaño relativo de ésta también varía según los deciles; en los hogares de los primeros estratos hay relativamente menos personas en edad de trabajar y proveer sustento económico. De este modo, aun cuando las tasas salariales, de ocupación y participación fueran iguales, los primeros estratos continuarían siendo, en términos relativos, menos acomodados. La contraparte, como se muestra en la sección siguiente, es una mayor presencia relativa de menores en los hogares más pobres.

En síntesis, entre los grupos de ingreso más bajo es menor la proporción de personas pertenecientes a la población en edad de trabajar que participan efectivamente en el mercado de trabajo, ya sea estando ocupados o bien buscando empleo. Esto limita el bienestar del hogar, pues aun cuando los salarios fuesen los mismos que en estratos más altos, la menor incorporación al mercado de trabajo aminora el total de ingresos que genera el hogar. Como se expone más adelante, la contrapartida de esta regularidad son mayores tasas de inactividad en los estratos de menores ingresos, particularmente entre los cónyuges.

\section{Tamaño y estructura de los hogares y su in- fluencia sobre la incorporación al mercado de trabajo}

Las dos últimas columnas del cuadro 4, así como el gráfico 1, ilustran respecto del tamaño de los hogares, apreciándose que sus miembros son más numerosos en los primeros deciles. Obsérvese también que los deciles superiores tienden a ser semejantes entre países. En los deciles inferiores, en contraste, las diferencias entre países son mayores. Con ciertas variaciones, en promedio el tamaño de hogar en los estratos más pobres excede en 50 a $60 \%$ aquel de los deciles superiores. Este es un primer indicio de que las diferencias entre países en la dinámica demográfica se deberían en gran parte a la medida en que los estratos de menores ingresos han reducido sus tasas de fecundidad. En contraste, los grupos de mayores ingresos de los diferen- 
GRÁFICO 1

América Latina (cinco países): Promedio de personas en el hogar, por deciles

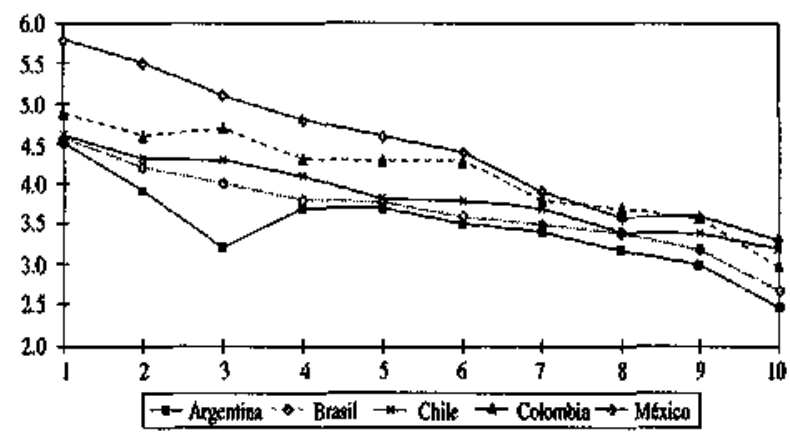

tes países parecen estar en una etapa de avance similar en la transición demográfica.

Como resultado de lo anterior, los hogares pobres se componen en mayor medida de menores, como muestra el gráfico 2, que ilustra la proporción de niños de hasta 11 años de edad por adulto en el hogar. Resalta la considerable diferencia entre los primeros deciles y los estratos superiores. Inclusive para países de avanzada transición demográfica, como Argentina y Chile, ${ }^{9}$ existen diferencias de 1 a 4 entre el primer decil y aquel superior en el promedio de niños por adulto en el hogar. Ello implica que la carga de cuidado de menores es considerablemente mayor entre los hogares más pobres, factor que a su vez se traduce en una alta incidencia de la inactividad y baja participación en el mercado de trabajo entre los cónyuges en estos estratos, como ilustra el gráfico 3.

Según se aprecia, en varios casos el porcentaje de cónyuges inactivos (de jefes ocupados) en los primeros deciles duplica aquel del decil más alto, reflejando una importante inequidad por estratos en el acceso efectivo al mercado de trabajo. En efecto, los grupos de menores ingresos enfrentan mayores costos de oportunidad e inferiores beneficios de incorporarse a este mercado. Por una parte, la mayor carga de cuidado de menores, en comparación con hogares de deciles más altos, incrementa el costo de oportunidad tanto de la búsqueda de empleo como de la incorporación a un empleo formal. Este problema es frecuentemente agravado por insuficiente disponibilidad o cobertura de servicios públicos y/o privados, en particular guarderías, jardines infantiles y escuelas. En segundo lugar, las remuneraciones asociadas a los bajos niveles educacionales alcanzados no compensan adecuadamente

\footnotetext{
${ }^{9}$ Véase en CELADE/BID (1996) un ordenamiento de países de la región según su avance en la transición demográfica.
}

GRÁFICO 2

América Latina (cinco países): Número de menores por adulto en el hogar, por deciles

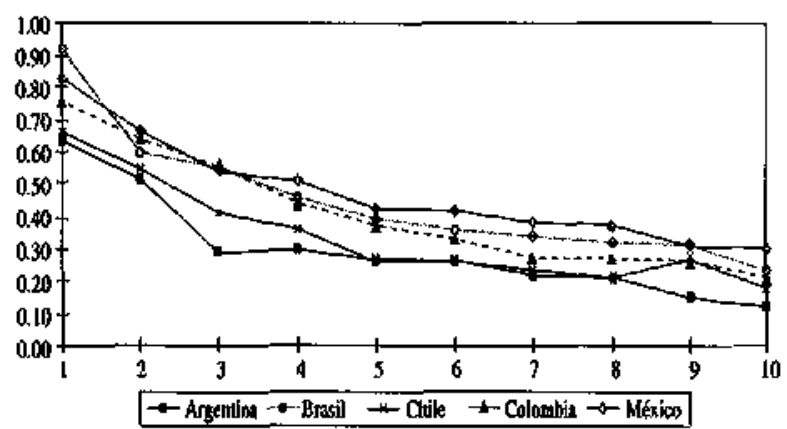

GRAFICO 3

América Latina (cinco países): Hogares en cada decil en los cuales el cónyuge es Inactivo, por deciles

(Porcentajes)

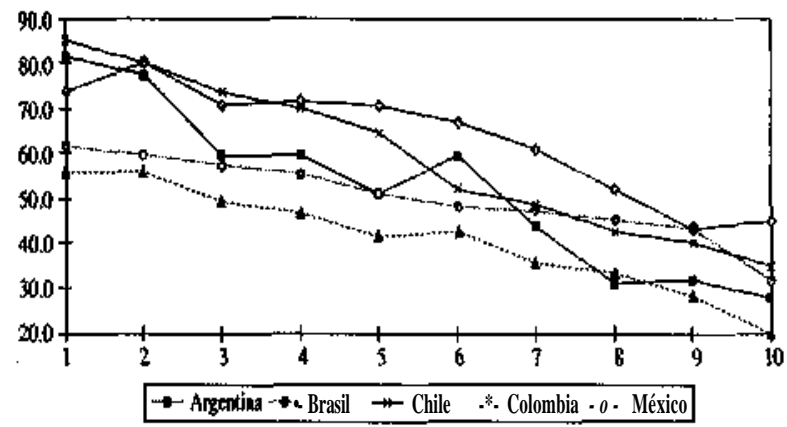

el costo en que se incurre. Este conjunto de factores induciría una menor incorporación al mercado de trabajo, especialmente al formal, por parte de los cónyuges, lo que se refleja en una alta incidencia de la inactividad y baja ocupación entre aquellos de los primeros estratos.

Esta situación ha sido persistente en el tiempo y en general ha tendido a agravarse, ampliándose las diferencias relativas. El cuadro 5 presenta, para dos puntos en el tiempo, el porcentaje de hogares ${ }^{10}$ en cada decil en los cuales ambos jefes están ocupados. Como allí se ve, persisten en el tiempo importantes diferencias. Se desprende de este cuadro, en primer lugar, que la proporción de hogares con ambos jefes ocupados crece con el nivel de ingreso en todos los casos. En segundo lugar, que en promedio se verifica un aumento de este porcentaje. En tercer lugar, que el incremento del porcentaje de hogares con ambos jefes ocupados

\footnotetext{
${ }^{10}$ El universo en cada decil se refiere a hogares en los cuales ambos jefes están presentes.
} 
CUADRO 5

América Latina (cinco países): Hogares en cada decíl con jefe y cónyuge ocupados como proporción del número de hogares en que ambos están presentes (Porcentajes)

\begin{tabular}{|c|c|c|c|c|c|c|c|c|c|c|}
\hline & \multicolumn{2}{|c|}{ Argentina' $^{\prime 1}$} & \multicolumn{2}{|c|}{ Brasil $^{\text {b }}$} & \multicolumn{2}{|c|}{ Chile $^{\mathrm{b}}$} & \multicolumn{2}{|c|}{ Colombia } & \multicolumn{2}{|c|}{ México $^{11}$} \\
\hline & 1980 & 1992 & 1979 & 1990 & 1987 & 1992 & $1980^{c}$ & $1992^{\mathrm{d}}$ & 1984 & 1992 \\
\hline Total & $1 S .5$ & 24.4 & 22.2 & 30.0 & 13.5 & 19.9 & 22.5 & 32.3 & 16.9 & 21.4 \\
\hline 1 & 2.9 & 3.1 & 21.5 & 20.9 & 2.0 & 2.8 & 10.1 & 14.6 & 10.1 & 15.1 \\
\hline 2 & 7.6 & 5.7 & 19.8 & 20.6 & 2.8 & 6.0 & 11.8 & 17.5 & 10.4 & 11.3 \\
\hline 3 & 10.1 & 10.2 & 18.2 & 24.8 & 4.5 & 9.8 & 12.6 & 25.7 & 14.9 & 18.4 \\
\hline 4 & 12.5 & 16.9 & 19.9 & 26.5 & 7.1 & 13,2 & 19.0 & 25,6 & 13.7 & 15,1 \\
\hline 5 & 13.0 & 22.8 & 18.3 & 28.1 & 10.0 & 16,8 & 19.5 & 32.7 & 13,1 & 17.0 \\
\hline 6 & 18.7 & 19.9 & 19.7 & 31.8 & 12.1 & 22.5 & 29.3 & 31.8 & 17.9 & 18.0 \\
\hline 7 & 25.1 & 33.8 & 21.6 & 33.1 & 16.5 & 27.4 & 28.0 & 35.9 & 18.8 & 23.6 \\
\hline 8 & 28.4 & 47.2 & 24.9 & 34.2 & 20.5 & 30.7 & 27.7 & 41.2 & 21.6 & 27.2 \\
\hline 9 & 35.5 & 49.6 & 27.7 & 37.7 & 27.5 & 34.4 & 31.9 & 45.2 & 22.5 & 36.4 \\
\hline 10 & 39.3 & 49.6 & 30.9 & 45.7 & 36.5 & 42.0 & 38.7 & 58.0 & 27.4 & 35.7 \\
\hline
\end{tabular}

Fuente: CEPAL sobre la base de tabulados especiales de encuestas de hogares.

${ }^{a}$ Buenos Aires. ${ }^{\mathrm{b}}$ Total nacional. ${ }^{\mathrm{c}}$ Siete ciudades principales. ${ }^{\mathrm{d}}$ Ocho ciudades principales.

entre el $30 \%$ más pobre es, en todos los países, inferior al aumento medio. Y en cuarto lugar, que el $30 \%$ superior de la distribución exhibe un alza mayor al promedio en esta variable en todos los países. En resumen, es desproporcionadamente menor el grado en que los cónyuges de los estratos inferiores se incorporan al mercado de trabajo y al empleo, y ocurre lo inverso en los estratos superiores." Con ello, los beneficios del crecimiento económico, del empleo, de las ganancias de productividad y los consiguientes incrementos en las remuneraciones, se circunscribirían a los deciles más altos de la distribución, en tanto que los restantes progresarían de un modo muy lento.

El mayor tamaño relativo de los hogares más pobres, así como la mayor presencia relativa de menores en ellos, no sólo tiene consecuencias sobre los niveles de participación en el mercado de trabajo, sino que también sobre los costos directos y de oportunidad para estos hogares de que jóvenes en edad de trabajar permanezcan adscritos al sistema educacional. Ello redunda en menores niveles educacionales entre los jóvenes y, por ende, menores remuneraciones futuras, reproduciendo en las siguientes generaciones una distribución concentrada děl ingreso.

En síntesis, la interacción de las características demográficas y los niveles educacionales y su efecto

\footnotetext{
11 Para comprobar esta hipótesis es preciso utilizar encuestas de corte longitudinal, las que sólo recientemente han comenzado a ser aplicadas en países de la región.
}

asociado sobre las remuneraciones configuran un cuadro que, a través del acceso diferenciado al mercado de trabajo, tiende a sesgar hacia los deciles superiores los beneficios del crecimiento económico. La inercia que esto origina se ve reflejada en un bajo avance en materia de distribución.

\section{Conclusiones}

Ciertamente existen factores determinantes de la distribución del ingreso que no se han examinado aquí, por el enfoque adoptado, por las limitaciones de espacio e información y por el propósito de centrar el análisis en ciertos factores respecto de los cuales es posible formular políticas. El enfoque empleado pone de relieve el modo en que se configura el ingreso del hogar, a diferencia de otros análisis que apuntan a explicar cómo influyen en el ingreso de los perceptores algunas características individuales (educación, género, experiencia, tipo de ocupación, entre otros) y los sectores a los que se adscriben. Algunos de estos aspectos han sido abordados aquí, pero el análisis ha hecho hincapié en las características de los hogares y sus miembros en su relación con la configuración del ingreso per cápita del hogar. Por otra parte, no fue examinado el diferente acceso que los estratos de ingreso tienen a acervos de capital, vivienda y servicios de salud, entre otros, que condicionan en grado importante el nivel de bienestar de las generaciones presentes y futuras. 
Entre los aspectos abordados, destacan las diferencias en cuanto a remuneración al trabajo, educación, tasas de ocupación, acceso al mercado de trabajo y factores demográficos que condicionan importantes desigualdades. Sumariamente, los principales resultados se enumeran a continuación.

En primer lugar, los ingresos factoriales medios de los grupos de menor ingreso per cápita son sustancialmente inferiores a los de estratos más altos, lo que se presenta asociado, en el caso de los ingresos provenientes del trabajo, a importantes diferencias en los niveles educacionales. Además, como otras estadísticas señalan, los sectores de menores ingresos se insertan en segmentos de baja productividad del mercado de trabajo. Se confirman de este modo los resultados obtenidos por estudios previos acerca de las desigualdades de ingreso entre perceptores.

En segundo lugar, las tasas de ocupación de los grupos más pobres son sistemáticamente inferiores al promedio. Los cambios en la distribución van asociados en gran medida a variaciones en los niveles de empleo, y las reducciones en las diferencias relativas de las tasas de ocupación se han presentado asociadas a una menor concentración del ingreso.
En tercer lugar, existen importantes diferencias entre deciles en el grado de participación en la fuerza de trabajo. Sistemáticamente se observan menores tasas de participación en los grupos menos acomodados. La contrapartida de ello son mayores tasas de inactividad, especialmente entre cónyuges y en un grado considerablemente menor, entre jefes de hogar.

En cuarto lugar, lo anterior se relaciona con la composición por edades de hogares en diferentes deciles. En efecto, según diversos indicadores la población en edad de trabajar es considerablemente menor en hogares más pobres. La proporción de niños por adulto es mayor en ellos, haciendo que soporten una mayor carga de cuidado de menores, y elevando así el costo de oportunidad de participar en el mercado de trabajo para las mujeres. ${ }^{12}$ Se suma este mayor costo a menores beneficios de participar en dicho mercado, factor que se relaciona con los niveles educacionales alcanzados.

Por último, es importante tener presente que ningún factor, considerado en forma aislada, logra dar cuenta completa de la distribución prevaleciente y sus cambios; sus efectos son más bien multiplicadores antes que aditivos.

\section{IV}

\section{Sugerencias de política}

De lo anterior se desprende que las estrategias orientadas a influir en la distribución del ingreso debieran actuar conjuntamente en cuatro áreas: i) generación de empleo productivo y aumento de la demanda de trabajo menos calificado; ii) políticas para aumentar y mantener los ingresos de los estratos más pobres; iii) reducción de las barreras a la incorporación al mercado de trabajo que éstos enfrentan, y iv) aspectos relacionados con la dinámica demográfica exhibida por estos segmentos.

Desde luego, la intensidad de la acción en cada esfera dependerá de la situación inicial de cada país. No obstante, debe recalcarse que el mejoramiento distributivo no se logra sólo mediante la acción sobre uno de los aspectos aquí señalados. En otras palabras, el objetivo de lograr una mayor equidad en la distribu-

\footnotetext{
12 Aun cuando el análisis estadístico se refiere a los cónyuges, en principio la segunda fuente de ingreso potencial para un hogar, la gran mayoría de estos son mujeres.
}

ción del ingreso excede los límites de las políticas de crecimiento, laborales y de población, siendo más bien el resultado del avance conjunto de ellas. En lo que sigue se sugieren políticas encaminadas a modificar en sentido progresivo la distribución del ingreso. También se examina la evolución de ciertas variables que permiten anticipar la evolución distributiva en el mediano plazo.

\section{Políticas de mediano y largo plazo en favor de una mejor distribución del ingreso}

a) Generación de empleo productivo y aumentos en
la demanda de trabajo menos calificado
$\mathrm{La}$ CEPAL ha desarrollado un planteamiento que consistentemente abarca los principales aspectos ligados a la distribución del ingreso (CEPAL, 1992 y 1997). En el corto plazo, se destaca la importancia de lograr elevados niveles de ocupación y la estabilidad macroeconómica, evitando así las consecuencias regresivas 
causadas por fluctuaciones del nivel de actividad debidas a procesos de ajuste como los experimentados por países de la región en los años ochenta. ${ }^{13}$ En una perspectiva de mediano y largo plazo dicho enfoque, entre otros aspectos, hace hincapié en la creación de empleo productivo a través del crecimiento económico sostenido como una de las condiciones básicas para lograr una mayor equidad en el tiempo. Acorde con ello, se proponen políticas de estímulo del ahorro y la inversión, así como la asignación eficiente de recursos, en el contexto de una mayor integración de las economías nacionales al comercio mundial. Entre estas políticas ocupa un lugar importante el fortalecimiento de las finanzas públicas, orientado a la generación de ahorro público para el financiamiento no inflacionario de los programas de inversión. Este esfuerzo público debiera completarse con el desarrollo de agentes de ahorro institucional, en particular de los sistemas de pensiones, y el establecimiento de un marco regulatorio que conduzca a un sistema financiero solvente que funcione con eficiencia y que canalice efectivamente el ahorro hacia la inversión real. Mantener un tipo de cambio real moderadamente alto y estable es crucial, no sólo como principal instrumento para promover los sectores exportadores y de sustitución de importaciones, sino que además, en conjunto con tasas reales de interés positivas y acordes con aquellas prevalecientes en los mercados mundiales, para mantener el ahorro financiero dentro del país y evitar así fugas especulativas de capitales. Se previenen de este modo fluctuaciones de los niveles de actividad, y su consiguiente impacto negativo sobre la inversión, que suelen derivarse de medidas de ajuste orientadas a enfrentar situaciones de desequilibrio externo. En suma, se propende al establecimiento de un marco de políticas orientadas al crecimiento, sobre la base de una mayor inserción externa y la recuperación de los niveles de inversión, apoyada principalmente en la generación de ahorro nacional.

No obstante, este enfoque reconoce que el mayor crecimiento no se traduce necesariamente en una expansión rápida de la demanda, por el tipo de trabajo ofrecido a los estratos de bajos ingresos, ni por ende en progreso distributivo. En razón de ello, también se delinean políticas orientadas al fomento de la pequeña

\footnotetext{
${ }^{13}$ Véase un examen de los efectos distributivos de los shocks y las políticas de corto plazo en Jiménez (1997). Allí se sintetizan resultados obtenidos mediante simulaciones, empleando modelos de equilibrio general para Argentina, Brasil y Colombia.
}

y mediana empresa, principales oferentes de empleo formal para los estratos de menores ingresos. Uno de los factores que limitan de un modo gravitante el desarrollo de estas empresas es la presencia de segmentaciones del mercado financiero que restringen el acceso al crédito. Como consecuencia, las posibilidades de inversión de éstas dependen estrechamente de la generación de excedentes internos, lo cual reduce su crecimiento y, con ello, la expansión de la demanda de trabajo menos calificado. Se necesitan nuevos instrumentos financieros que superen la carencia de garantías reales en estas empresas, acompañados por programas de cooperación técnica y apoyo público al. mejoramiento de su capacidad de gestión y adopción de progreso tecnológico, para liberar el potencial de crecimiento de pequeñas y medianas empresas y, con ello, de la demanda de trabajo (Held, 1995).

Desafortunadamente, la evolución en materia de ahorro, inversión y crecimiento en la región entre 1990 y 1996 fue insuficiente para inducir aumentos significativos en la generación de empleo (CEPAL, 1997). Entre 1991 y 1996, sólo cuatro países de la región lograron un crecimiento anual medio estable superior al $4 \%$. Otros países lograron ocasionalmente tasas mayores, pero sin lograr sostenerlas. En suma, además de moderado (3.1\% como promedio entre 1991 y 1996), el crecimiento en la región ha sido inestable, factor que ha gravitado en la baja expansión del empleo y las alzas en el desempleo (CEPAL, 1997). Por otra parte, empleando un indicador compuesto por cinco variables (desempleo, informalidad, salarios en la industria, salarios mínimos y productividad) la Organización Internacional del Trabajo (OIT) señala que, en una muestra de 16 países de la región, sólo cinco (Bolivia, Chile, Panamá, Paraguay y Perú), exhibieron un progreso laboral tendencial positivo entre 1990 y 1996, y únicamente Chile presentó avances en todas las variables, incluida la productividad; en los once países restantes, entre los cuales se hallan las economías más grandes y populosas de América Latina y el Caribe, se observaron retrocesos o estancamientos de carácter tendencial en los índices de progreso laboral (OIT, 1996). Como resultado de lo anterior, salvo excepciones, no es posible anticipar mejoramientos significativos de la distribución del ingreso en la región. Por el contrario, debido a la influencia de la variable ocupacional sobre la distribución, así como por la mayor incidencia del desempleo entre los estratos más pobres, lo más probable es que aumente la concentración del ingreso en un número importante de países. 
b) Incremento y mantención de los ingresos de los hogares más pobres: educación y transferencias Como se señaló, el nivel educacional afecta en medida importante la remuneración de quienes logran empleo, por lo que las medidas para promover la ampliación de la cobertura del sistema educacional, el incremento de sus tasas de retención y las mejoras en su calidad, forman parte de toda política que se oriente a mejorar la distribución del ingreso en el largo plazo. Sin embargo, en términos distributivos el resultado de estos esfuerzos sólo cristalizará a largo plazo, a medida que sucesivas cohortes con mejores niveles de educación se incorporen al empleo y adquieran importancia numérica. Los programas de capacitación orientados a elevar el nivel de calificación de aquellos más pobres, probablemente exhiban un resultado más cercano, a mediano plazo. Además, estos programas pueden reforzar la eficacia de las políticas de largo plazo, por cuanto existen complementariedades intergeneracionales. Por ejemplo, alzas actuales en el ingreso del hogar, como resultado de mejoras salariales asociadas a programas de capacitación, aumentarían la permanencia escolar de los hijos, evitando su prematura incorporación a empleos de baja remuneración. Con ello, en el futuro éstos podrán aspirar a mejores ingresos.

También existen importantes relaciones de complementariedad con políticas de otras esferas, como es el caso de la provisión de servicios de salud a la población pobre; al mejorar ésta, se refuerzan los efectos de la política de educación, pues permite mayor continuidad en la permanencia dentro del sistema escolar y un mejor aprovechamiento de éste por los menores. A su vez, como lo han demostrado varios estudios, los mayores niveles de educación de las madres se traducen, entre otras cosas, en menores tasas de morbilidad y mortalidad infantil (CELADE/BID, 1996), En ambos casos, los resultados distributivos son de larga maduración. Por ello, es clave la persistencia de estas políticas y del crecimiento económico ya que las reducciones transitorias de los recursos destinados a ellas generan efectos permanentes. Quienes hoy, por ejemplo, pierden la oportunidad de permanecer en el sistema educativo por una reducción transitoria de ingresos en el hogar, encontrarán dificultades para reincorporarse en el futuro, con lo cual su calificación y por ende sus ingresos podrían ser permanentemente más bajos. Las oscilaciones negativas en los recursos destinados a la política social tienden entonces a reforzar los mecanismos intergeneracionales de transmisión de la pobreza.

La experiencia de la región en este ámbito tampoco permite anticipar, con algunas excepciones, una evolución progresiva de la distribución. Como lo señalan diversos estudios, frecuentemente fueron los recursos destinados a las políticas sociales los que experimentaron las mayores reducciones reales debido a ajustes del presupuesto público (CEPAL, 1994b; Cominetti, 1994), agravando con ello efectos distributivos regresivos derivados de la recesión. Por lo tanto, la falta de progresos distributivos en los años noventa puede atribuirse en parte a etapas de ajuste macroeconómico vividas por la mayoría de los países de la región en los años ochenta.

El análisis precedente se refiere a políticas que de un modo indirecto, aunque determinante, intentan afectar el nivel de ingreso de quienes consiguen un empleo y, por lo tanto, la distribución. Otras políticas de intervención directa también son importantes. El establecimiento de salarios mínimos es una de las prácticas más difundidas, aunque existen apreciaciones divergentes respecto de su eficacia y conveniencia.

En términos generales habría cierto consenso en estimar que los incrementos salariales que sobrepasan los aumentos de productividad tienen efectos distributivos poco claros, pero por lo general negativos, para los niveles de actividad. ${ }^{14}$ En cuanto a los salarios mínimos, hay bases para creer que las pérdidas reales en ellos se asocian a incrementos en la incidencia de la pobreza y viceversa, aunque el efecto en términos del coeficiente de Gini no sea discernible. Por ello, en tanto el crecimiento real de los salarios mínimos no sea mayor que el de la productividad media de la economía, ${ }^{15}$ cabe anticipar que reduciría la incidencia de la pobreza, con lo cual continúa siendo una importante herramienta de política (Jiménez, 1996; Morley, 1997).

Desafortunadamente, durante la primera mitad de los años noventa los salarios mínimos reales urbanos evolucionaron de un modo tal que, al menos por esta vía, no se vislumbran mejorías en materia distributivas. Así, según señala la OTT (1996) respecto a una muestra de 18 países de la región, sólo en cuatro (Colombia, Costa Rica, Panamá y Paraguay) los salarios mínimos reales urbanos en 1995 excedieron el nivel de 1980; aún más, sólo en Costa Rica el crecimiento real de esta variable fue de cierta significación; como resultado, en promedio para la región el nivel real de estos salarios

\footnotetext{
14 Véanse Chisari y Romero (1996), Urani, Moreira y Willcox (1997), Lora y Fernández (1996) y Jiménez (1997).

${ }^{15}$ Téngase presente que el aumento de la productividad de las pequeñas empresas y sectores informales, importantes requirentes de trabajo menos calificado, suele ser inferior a la del resto de ia economía.
} 
mínimos en 1995 equivale a un 72\% del de 1980, habiendo crecido a una tasa casi nula, de $0.8 \%$ anual, entre 1990 y 1995.

Una segunda alternativa para modificar la distribución del ingreso son las transferencias directas, que se componen principalmente de pensiones, jubilaciones y subsidios. El propósito del análisis que sigue no es agotar las opciones de mecanismos de transferencias y su contenido redistributivo, sino que más bien señalar que, dado el carácter indirecto y de largo plazo que revisten las políticas orientadas a modificar en sus fuentes la generación del ingreso, ciertas acciones tienen un importante potencial de distribución progresiva a plazo más cercano, lo que debe tenerse en cuenta. En la región se han dado numerosas experiencias de transferencias de ingresos y subsidios cuyo objetivo ha sido paliar los efectos de determinados factores que causan pobreza y distribución regresiva. Es pertinente hacer ciertos alcances breves a la eficiencia y eficacia de algunas de las principales modalidades de transferencias.

Con frecuencia se alude a posibilidades de redistribuir ingresos a partir de la utilización de los sistemas de pensiones y jubilaciones. Es apropiada aquí una importante digresión. En primer lugar, como ha sido ampliamente respaldado por estudios de casos nacionales, uno de los principales factores que originaron la precaria situación de estos sistemas en algunos países ha sido la falta de correspondencia entre aportes y beneficios, lo que trajo consigo una baja identificación con los propósitos de éstos, e incentivó la subdeclaración y la evasión. ${ }^{16}$ Una característica relevante de las reformas recientes a los sistemas de pensiones es la tendencia a vincular más las contribuciones y los beneficios, como manera de promover su solvencia. Implícitamente, se ha reconocido de este modo que la introducción de criterios redistributivos en los sistemas de pensiones puede ejercer una considerable influencia negativa sobre su eficiencia, solvencia y estabilidad financiera. En suma, estos sistemas serían un mecanismo ineficiente de redistribución.

En segundo lugar, en la experiencia regional la cobertura de los sistemas de pensiones suele ser insuficiente, de modo que si se efectúa una acción redistributiva a través de ellos, probablemente se excluya a una parte mayoritaria de los estratos más pobres. En otras palabras, la eficacia de estos sistemas como me-

\footnotetext{
${ }^{16}$ Véanse Iglesias y Acuña (1991); Uthoff y Szalachman, comps (1991, 1992 y 1994); Schulthess y Demarco (1993), y Uthoff (1995).
}

canismos de redistribución progresiva estaría lejos de ser la esperada, puesto que su alcance es inferior al necesario para llegar a los grupos deseados. No obstante, los sistemas de pensiones que incluyen un componente de financiamiento solidario de hecho tienen un potencial redistributivo hacia segmentos medios y bajos incluidos en su cobertura, que podría ser aprovechado en la medida en que se confronten los problemas de solvencia.

Por otra parte, la actual orientación de las reformas a los sistemas de pensiones no invalida políticas de carácter redistributivo como las de proveer una pensión mínima a quienes, a pesar de sus contribuciones al sistema, no alcancen cierto nivel de ingreso al momento de su retiro. Más aún, los perfiles de pobreza señalan una mayor incidencia de ella entre los pensionados. En consecuencia, cumplen una importante función distributiva y paliativa de la pobreza las medidas tendientes a proveer pensiones mínimas, así como otras de carácter asistencial (ligadas, por ejemplo, a accidentes, enfermedad y viudez), unidas a la ampliación de la cobertura del sistema de pensiones y en general de las redes de protección social.

Un segundo tipo de transferencias, cuyo examen ha suscitado cierto interés recientemente, es el de las vinculadas a la mayor incidencia del desempleo en los hogares pobres. En el cuadro 6 se presenta la distribución de los desempleados por deciles.

Como se aprecia en el cuadro, los desocupados se concentran desproporcionadamente en los primeros deciles de ingresos, aun cuando los países incluidos atravesaban por diferentes fases del ciclo en los años considerados. Dos situaciones de desempleo, que originan políticas distintas, deben ser tenidas en cuenta para explicar lo anterior. En primer lugar, la considerable mayor incidencia del desempleo en el primer decil se debería en parte a factores causantes de desempleo crónico, que ni las recuperaciones del crecimiento ni su mantención en el tiempo logran reducir significativamente. Se trata en este caso del núcleo duro de la pobreza. Paliar la incidencia de ésta requeriría, entre otras cosas, programas de empleo mínimo, cuya duración excediera la de programas de emergencia y que proveyeran un nivel básico de remuneración, aunque no deberían convertirse en un desincentivo a la incorporación de estos desocupados al mercado de trabajo en pos de empleos de productividad y remuneración mayores. El segundo tipo de desempleados se asocia más cercanamente al ciclo económico, incidiendo también en mayor medida en los grupos más pobres. Las transferencias, o bien los programas de em- 
CUADRO 6

América Latina (cinco países): Distribución de los desocupados, por deciles (Porcentajes)

\begin{tabular}{|c|c|c|c|c|c|}
\hline & $\begin{array}{c}\text { Argentina, } \\
1992^{\mathrm{a}}\end{array}$ & $\begin{array}{c}\text { Brasil, } \\
1990^{\mathrm{b}}\end{array}$ & $\begin{array}{c}\text { Colombia, } \\
1992^{\mathrm{c}}\end{array}$ & $\begin{array}{l}\text { Chile, } \\
1992^{\text {d }}\end{array}$ & $\begin{array}{c}\text { México, } \\
1992^{\mathrm{C}}\end{array}$ \\
\hline Total & 100 & 100 & 100 & 100 & 700 \\
\hline 1 & 33.6 & 25.6 & 18.6 & 29.0 & 13.8 \\
\hline 2 & 19.2 & 13.1 & 15.4 & 15.3 & 17.0 \\
\hline 3 & 9.8 & 12.2 & 11.9 & 14.4 & 15.2 \\
\hline 4 & 14.0 & 13.0 & 11.0 & 9.2 & 10.2 \\
\hline 5 & 7.8 & 9.6 & 10.6 & 9.7 & 11.3 \\
\hline 6 & 5.1 & 6.7 & 11.0 & 5.6 & 7.4 \\
\hline 7 & 5.2 & 7.1 & 6.2 & 5.8 & 10.8 \\
\hline 8 & 0.9 & 5.6 & 7.0 & 3.9 & 2.5 \\
\hline 9 & 2.1 & 3.4 & 5.4 & 4.9 & 7.5 \\
\hline 10 & 2.4 & 3.5 & 2.7 & 2.2 & 4.2 \\
\hline
\end{tabular}

Fuente: CEPAL, sobre la base de tabulados especiales de encuestas de hogares.

" Buenos Aires. $\quad{ }^{\mathrm{b}}$ Sao Paulo y Río de Janeiro. ${ }^{\mathrm{c}}$ Bogotá. ${ }^{\mathrm{d}}$ Gran Santiago. ${ }^{\mathrm{c}}$ Zonas de alta densidad.

pieos de emergencia, orientados a reemplazar ingresos en situaciones transitorias de desempleo contribuirían a evitar que aumente la concentración del ingreso durante períodos recesivos.

En la eficacia y eficiencia de estos esquemas influyen mucho las modalidades de financiamiento (seguros de desempleo con aportes individuales, fondos públicos, etc.), los criterios con que se eligen los beneficiarios, la duración del beneficio y el grado en que se reemplaza el ingreso perdido. Como no es posible efectuar aquí un análisis detallado, estas líneas más bien destacan que tales herramientas son importantes porque contribuirían a conjurar, al menos parcialmente, la agudización de la pobreza y la concentración del ingreso que suelen acarrear períodos de ajuste macroeconómico.

Dichas herramientas, de carácter principalmente compensatorio o paliativo, pueden ser incorporadas productivamente a una política global tendiente a garantizar un nivel de ingreso familiar mínimo, lo que permitiría superar algunas deficiencias que por sí solas ellas exhiben (CEPAL, 1992). En efecto, como se ha señalado, las políticas de salarios mínimos tienen un alcance parcial, restringido principalmente a sectores urbanos formales, a la vez que afectan en mayor grado la rentabilidad de pequeñas empresas, principales demandantes de trabajo menos calificado. Aun más, en el caso de recesiones por insuficiencia de demanda interna su eficacia es limitada. En consecuencia su nivel no puede ser muy elevado ni tampoco puede ser la única herramienta que se use. Similares problemas de cobertura presentan las pensiones mínimas y asistenciales y los esquemas de seguros de desempleo y subsidios de cesantía. Ello lleva a pensar en la utilización de un conjunto de herramientas orientadas a lograr cierto nivel mínimo de ingreso familiar, mediante una combinación de salarios y pensiones mínimas, subsidios y/o seguros de desempleo, más asignaciones familiares y/o bonos o transferencias directas o en especie distribuidas, por ejemplo, a través del sistema escolar público. En el caso de que no se alcance el nivel de ingreso familiar mínimo estipulado, estas medidas se suplementarían con transferencias asignadas según la incidencia de los factores que determinan la severidad de la pobreza y la concentración del ingreso. Por cierto, para llevar a cabo lo descrito es preciso establecer métodos de evaluación de la situación de ingresos y/o de carencia de cada familia a partir de registros especiales, como se ha hecho algunas veces en la región. ${ }^{17}$

Finalmente, cabe hacer un alcance general al tema del financiamiento de transferencias de tipo redistributivo o bien orientadas a paliar el efecto de factores que inciden sobre la severidad de la pobreza. El efecto final de ellas sobre la distribución del ingreso depende también de los efectos del método de financiamiento del gasto público. Parte importante de las

\footnotetext{
${ }^{17}$ Un ejemplo es la operación del Subsidio Único Familiar (sur), en Chile, basado en ¡a ficha CAS, de caracterización socioeconómica, que considera diversos factores para establecer la elegibilidad de un grupo familiar para recibir este subsidio.
} 
CUADRO 7

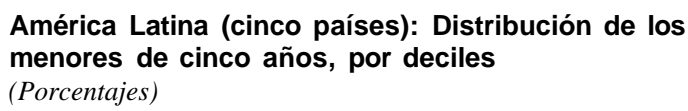

\begin{tabular}{|c|c|c|c|c|c|}
\hline & $\begin{array}{c}\text { Argentina, } \\
1992^{\mathrm{a}}\end{array}$ & $\begin{array}{c}\text { Brasil, } \\
1990^{b}\end{array}$ & $\begin{array}{c}\text { Colombia, } \\
1992^{\mathrm{C}}\end{array}$ & $\begin{array}{l}\text { Chile, } \\
1992^{\mathrm{a}} \\
\end{array}$ & $\begin{array}{c}\text { México, } \\
1992^{\mathrm{C}}\end{array}$ \\
\hline Total & 100 & 100 & 100 & 100 & 100 \\
\hline 1 & 22.6 & 20.4 & 18.4 & 19.3 & 17,2 \\
\hline 2 & 18.7 & 14.6 & 15.4 & 17.0 & 12,5 \\
\hline 3 & 10.9 & 12.6 & 14.7 & 12.6 & 13.2 \\
\hline 4 & 10.5 & 10,8 & 10.8 & 10.5 & 11.2 \\
\hline 5 & 7.9 & 8.9 & 8.9 & 8.0 & 11,2 \\
\hline 6 & 7.8 & 7.9 & 7.7 & 9.0 & 8.8 \\
\hline 7 & 6.9 & 7.7 & 6.3 & 6.4 & 7.5 \\
\hline 8 & 7.0 & 7.4 & 7.3 & 5.7 & 6.4 \\
\hline 9 & 4.0 & 5.7 & 5.6 & 6.5 & 5.9 \\
\hline 10 & 3.7 & 4.0 & 4.8 & 5.0 & 6.2 \\
\hline
\end{tabular}

Fuente: CEPAL, sobre la base de tabulados especiales de encuestas de hogares.

${ }^{a}$ Buenos Aires. ${ }^{b}$ Sao Paulo y Río de Janeiro. ${ }^{c}$ Bogotá. $\quad{ }^{\text {d }}$ Gran Santiago. ${ }^{c}$ Zonas de alta densidad.

reformas tributarias introducidas recientemente en la región se ha orientado a elevar la recaudación, así como también a mejorar aspectos de eficiencia del sistema tributario, ampliando la participación o bien introduciendo tributos indirectos, como el impuesto al valor agregado. Si bien hay muchas razones para tomar tal camino, debe tenerse en cuenta la regresividad de este método de financiamiento. En efecto, como se desprende de varios estudios de casos, financiar incrementos del gasto público mediante impuestos indirectos es, desde el punto de vista estrictamente distributivo, la opción menos deseable, ${ }^{18}$ porque hay más posibilidades de traslación de su incidencia que en el caso de los impuestos directos. En consecuencia, desde esta perspectiva, es deseable que las futuras reformas eleven la carga tributaria directa antes que la indirecta.

c) Reducción de las barreras que enfrentan los más pobres para participar en el mercado de trabajo Como se discutió más arriba, el costo de oportunidad de participar en el mercado de trabajo que enfrentan los hogares de menores ingresos es más alto, por la mayor presencia relativa de menores en el hogar. Como se aprecia en el cuadro 7, en el $30 \%$ más pobre de los hogares se concentra la mayor parte de los niños menores de cinco años, llegando en algunos

\footnotetext{
${ }^{18}$ Véanse Chisari y Romero (1996), Lora y Fernández (1996), Urani, Moreira y Willcox (1997) y Jiménez (1997).
}

casos al 50\%. ${ }^{19}$ Por lo tanto, una política de desarrollo de instancias institucionales para el cuidado de menores, ya sean éstas privadas o públicas, favorecería la incorporación de los cónyuges al mercado de trabajo, al reducir uno de los factores que causan una distribución concentrada del ingreso, como es la menor tasa de participación relativa y las tasas considerablemente mayores de inactividad entre los cónyuges de hogares pobres.

Es importante tener presente ciertos factores que condicionan el éxito de lo anteriormente propuesto. En primer lugar, no sólo es mayor el costo de oportunidad de participar en el mercado laboral para los cónyuges pertenecientes a hogares pobres, sino que, por las bajas remuneraciones que se originan en bajos niveles de calificación, también es poco el incentivo que tienen para una mayor participación. Por lo tanto, junto con tomar medidas para reducir las barreras que dificultan el acceso al mercado laboral de los cónyuges pertenecientes a hogares de menores ingresos, es preciso establecer programas de capacitación orientados específicamente a estas personas. En segundo lugar, las consideraciones y medidas anteriores están vinculadas principalmente a la oferta, y su resultado depende del aumento de la demanda de trabajo merced al crecimiento económico y del incentivo a secto-

\footnotetext{
${ }^{19}$ Esto implica además que las transferencias en efectivo, servicios o especies cuyo criterio de asignación tome en cuenta la presencia de niños en el hogar pueden tener un alto contenido distributivo.
} 
res que requieren trabajo menos calificado, como las pequeñas y medianas empresas. En este sentido se dijo antes que sólo a través de la acción conjunta en Jas áreas mencionadas es posible lograr mejoramientos distributivos duraderos.

Un segundo segmento del mercado de trabajo que tiene problemas de acceso es el de los jóvenes, entre los cuales la incidencia del desempleo es característicamente más elevada. La falta de calificación figura en este caso como uno de los factores gravitantes. Actualmente en la región se dan varios intentos de promover una mayor participación de los organismos de productores privados en el diseño y ejecución de los programas de capacitación, para lograr una mayor coincidencia entre la demanda de calificaciones y el contenido de esos programas (CEPAL/UNESCO, 1996). Se aspira a que quienes egresan de las instituciones de capacitación puedan acceder de modo más expedito a un empleo, para lo cual se busca superar el esquema pasivo en el cual las instituciones de capacitación enseñan sobre la base de sus capacidades tradicionales, que pueden no responder a la demanda, y dejan el acceso al empleo totalmente por cuenta de quienes asisten a estos programas.

Puesto que la falta de calificaciones entre los jóvenes es un obstáculo para su acceso al mercado de trabajo, debe tenerse presente que los estratos más pobres en muchos casos enfrentan serias dificultades para mantener a los jóvenes en el sistema escolar, incluida la enseñanza media, y luego en instituciones de capacitación. Por este motivo, la flexibilización curricular de la enseñanza también debe considerarse un mecanismo tendiente a dar formación técnico-profesional más temprana a personas que no deseen o no estén en condiciones de ingresar a la educación superior.

\section{d) Aspectos relacionados con la dinámica demográ- fica}

Como se ha recalcado más atrás, los aspectos demográficos influyen en grado importante sobre la situación distributiva, incluso en países como Argentina y Chile, que ya han avanzado en la transición demográfica hacía menores niveles de fecundidad. Los estratos más pobres, como se señaló antes, se caracterizan por exhibir hogares con mayor número de personas y mayor presencia relativa de menores y, en consecuencia, menos integrantes en edad de trabajar.

Diversos estudios demuestran que el avance en la transición demográfica ha sido más pronunciado en los estratos de ingreso más alto; los grupos pobres, en cambio, aún exhiben tasas de fecundidad altas, consi- derablemente superiores al promedio (CELADE/BID, 1996). Los estratos más pobres son también los que menos regulan su fecundidad, siendo menos espaciados los embarazos; hechos ambos que, sin mencionar otras consecuencias negativas, se traducen en una mayor inestabilidad de la participación de la mujer en el mercado laboral.

Además, como ha sido ampliamente documentado, los grupos más pobres exhiben una mayor proporción de fecundidad no deseada; en otras palabras, hubieran preferido tener menos hijos en una proporción mayor que los grupos que disfrutan de más bienestar. ${ }^{20}$ La educación de la madre ha probado ser una variable que influye mucho en esta situación; mientras menor es su nivel de instrucción, mayor es la fecundidad observada y mayor es la proporción de ésta que es no deseada ex-post. En contraste, las madres con mayores niveles de instrucción exhiben menor fecundidad, y una proporción inferior de ésta es no deseada. Lo dicho pone de manifiesto una importante diferencia en el acceso a la planificación familiar entre estratos socioeconómicos, que se traduce en inequidad para ejercer un control efectivo sobre las decisiones que afectan a la fecundidad. En efecto, según se desprende de estudios especiales, las madres con menos instrucción recurren en mayor medida a métodos tradicionales de control del número de embarazos o bien no los emplean en absoluto, en tanto que las madres con mayor nivel de instrucción recurren en mayor proporción a métodos modernos (ibid.). En consecuencia, las políticas que faciliten el acceso a la planificación familiar y que hagan hincapié ex ante en la paternidad responsable, son parte necesaria de las estrategias orientadas a una mayor equidad y mejor distribución del ingreso.

En segundo lugar, como se señaló antes, la eficacia de políticas de largo aliento como la de educación, depende en gran medida de las condiciones de salud en que se encuentran quienes ingresan al sistema educacional. En este aspecto son determinantes la salud de la madre durante el período de gestación y el estado de salud y nutrición de los niños durante sus primeros años. Desde esta perspectiva también forman parte clave de una estrategia distributiva los programas de salud y nutrición materno-infantil, por cuanto sin que éste sea su objetivo principal, contribuyen a crear condiciones para que en el largo plazo los grupos más pobres eleven sus calificaciones e ingresos. A su vez, el grado de instrucción de la madre es una variable que

\footnotetext{
${ }^{30}$ Véanse CEPAL (1992), CELADE/BID (1996) y CEPAL/CELADE (1998).
} 
incide en gran medida en la mortalidad infantil. Ha sido suficientemente documentado (ibíd.) que las tasas de mortalidad y morbilidad infantil son menores entre los hijos de madres con mayor educación. Nuevamente, lo anterior demuestra que una política redistributiva debe actuar en varios frentes a la vez, reconociendo de este modo la multiplicidad de los factores que determinan la distribución del ingreso.

Las políticas que se han mencionado tienen contenido tanto de equidad como de redistribución de ingresos. Ellas contribuyen a reforzar en el tiempo el efecto de medidas encaminadas a aumentar la generación de ingresos entre los más pobres. Si en virtud de estas políticas se reduce la fecundidad en los estratos más rezagados, acortando la distancia entre el número de hijos deseado y el efectivo, tenderá a aminorarse la mayor carga relativa de cuidado de menores en esos estratos. Esto posibilitaría una mayor participación de los cónyuges en el mercado laboral, y si se llevan a cabo programas de capacitación adecuados sus ingresos podrían verse mejorados.

Por otra parte, cuanto menor sea el número de niños, más disminuirán los recursos necesarios para mantenerlos en el sistema escolar y se reducirá la presión para abandonarlos en busca de trabajo con el fin de entregar sustento a la familia. Ambas cosas contribuyen a prolongar la permanencia de los menores en el sistema escolar, los que podrán aspirar a mejores ingresos en el futuro.

No obstante, para que estos cambios demográficos ocurran se necesita una acción deliberada que promueva un mayor acceso a la planificación familiar y también medidas eficaces para reforzar la generación de ingresos entre los más pobres. De este modo, los cambios serán también el resultado de la voluntad de los estratos más pobres, que adoptarán una actitud distinta respecto de su fecundidad, en la confianza de que con ello contribuirán a su propio bienestar futuro.

\section{Bibliografía}

Altimir, O. (1994): Distribución del ingreso e incidencia de la pobreza a lo largo de! ajuste. Revista de la CEPAL, $\mathrm{N}^{\circ} 52$, LC/G.1824-P, Santiago de Chile, Comisión Económica para América Latina y el Caribe (CEPAL),

Altimir, O. y S. Piñera (1977): Análisis de descomposición de las desigualdades de los ingresos primarios en países de América Latina, Santiago de Chile, CEPAL.

CELADE/BID (Centro Latinoamericano de Demografía/Banco Interamericano de Desarrollo) (1996): Impacto de las tendencias demográficas sobre los sectores sociales en América Latina, Santiago de Chile.

CEPAL (1991): Magnitud de la pobreza en América Latina en los años ochenta. Estudios e Informes de la CEPAL, $\mathrm{N}^{\circ} 81$ LC/G.1653-P, Santiago de Chile. Publicación de las Naciones Unidas, $\mathrm{N}^{\circ}$ de venta S.91.II.G.10.

(1992): Equidad y transformación productiva: un enfoque integrado, LC/G.1701/Rev,1-P, Santiago de Chile, Publicación de las Naciones Unidas, $\mathrm{N}^{\circ}$ de venta S.92.II.G.5..

(1994a): Panorama social de América Latina, ;994, LC/G.1844, Santiago de Chile.

(1994b): El gasto social en América Latina. Un examen cuantitativo y cualitativo, Cuadernos de la CEPAL, $\mathrm{N}^{\circ} 73$, LC/G.1854-P, Santiago de Chile, CEPAL.

(1995): Panorama social de América Latina, 1995,

LC/G.1886-P, Santiago de Chile.

(1996a): Panorama social de América Latina, 1996,

LC/G.1946-P, Santiago de Chile.

(1996b): La sensibilidad del indicador de pobreza. Un análisis a partir de diferentes opciones metodológicas, LC/R1657, Santiago de Chile, CEPAL.

. (1997): La brecha de equidad. América Latina, el Caribe y la cumbre social LC/G.1954/Rev.1-P, Santiago de Chile, Publicación de las Naciones Unidas, $\mathrm{N}^{\circ}$ de venta S.97.II.G.11.

CEPALCELADE (Comisión Económica para América Latina y el Caribe/Centro Latinoamericano de Demografía) (1998):
América Latina: la transición demográfica en sectores rezagados, CELADE, Santiago de Chile,

CEPAL/UNESCO (Comisión Económica para América Latina y el Caribe/Organización de las Naciones Unidas para la Educación, la Ciencia y la Cultura) (1996): Educación y conocimiento, eje de ia transformación productiva con equidad, Santiago de Chile.

Chisari, O. y C. Romero (1996): Distribución del ingreso, asignación de recursos y shocks macroeconómicos. Un modelo de equilibrio general computado para la Argentina en 1993, serie Financiamiento del desarrollo, $\mathrm{N}^{\mathrm{o}} 36$, Santiago de Chile, CEPAL.

Cominetti, R. (1994): Gasto social y ajuste fiscal en América Latina, serie Reformas de política pública, $\mathrm{N}^{\circ} 20$, Santiago de Chile, CEPAL.

Feres, J. C. (1995): La medición de los ingresos en ja perspectiva de los estudios de pobreza. El caso de la Encuesta Casen de Chile: años 1987 a 1994, LC/R.1604, Santiago de Chile, CEPAL.

Held, G. (1995): Políticas de financiamiento de las empresas de menor tamaño: experiencias recientes en América Latina, serie Financiamiento del desarrollo, $\mathrm{N}^{\circ} 34$, Santiago de Chile, CEPAL.

Iglesias, A. y R. Acuña (1991): Chile: experiencia con un régimen de capitalización 1981-1991, Santiago de Chile, CEPAL/Programa de las Naciones Unidas para el Desarrollo (PNUD).

Jiménez, L. F. (1996): La experiencia de ajuste durante la década de los ochenta en Latinoamérica, consecuencias distributivas y diseño de políticas sociales, Comisión Económica para América Latina y el Caribe/Centro Latinoamericano de Administración para el Desarrollo/Sistema Económico Latinoamericano (CEPAL/CLADES/SELA), Desarrollo con equidad. Hacia una nueva articulación de políticas económicas y sociales en América Latina y el Caribe, Caracas, Nueva Sociedad. (1997): Distribución del ingreso, shocks y políticas macroeconómicas, serie Financiamiento del desarrollo, $\mathrm{N}^{\circ} 44$, Santiago de Chile, CEPAL. 
Jiménez, L. F. y N. Ruedi (1997a): Algunos factores que inciden en la distribución del ingreso en Argentina, 1980-1992. Un análisis descriptivo, serie Financiamiento del desarrollo, $\mathrm{N}^{\circ}$ 67, Santiago de Chile, CEPAL.

(1997b): Algunos factores que inciden en la distribución del ingreso en Colombia, 1980-1992, Un análisis descriptivo, serie Financiamiento del desarrollo, $\mathrm{N}^{\circ} 68$, Santiago de Chile, CEPAL.

(1997c): Algunos factores que inciden en la distribución del ingreso en Chile, 1987-1992, Un análisis descriptivo, serie Financiamiento del desarrollo, $\mathrm{N}^{\circ} 69$, Santiago de Chile, CEPAL Naciones Unidas, Santiago, Chile.

(1997d): Un análisis descriptivo de la distribución del ingreso en México, 1984-1992, serie Financiamiento del desarrollo, $\mathrm{N}^{\circ} 70$, Santiago de Chile, CEPAL.

. (1997e): Un análisis descriptivo defactores que inciden en la distribución del ingreso en Brasil, 1979-1990, serie Financiamiento del desarrollo, $\mathrm{N}^{\circ} 71$, Santiago de Chile, CEPAL.

Lora, E. y C. Fernández (1996): Efectos de los shocks macroeconómicos y de las políticas de ajuste sobre la distribución del ingreso en Colombia, serie Financiamiento del desarrollo, $\mathrm{N}^{\circ} 38$, Santiago de Chile, CEPAL.

Morley, S. (1997): Poverty during recovery and reform in Latín America: 1985-1995, Washington, D.C., BID, inimeo.

OIT (Organización Internacional del Trabajo) (1996): Panorama Laboral 1996, Lima, Oficina Regional de la OIT para América Latina y el Caribe.

Ocampo, J.A., M.J. Pérez, C. Tovar y F.J. Lasso (1998): Macroeconomía, ajuste estructural y equidad en Colombia, 1978¡996, Archivos de macroeconomía, N 79, Santafé de Bogo- tá, República de Colombia, Departamento Nacional de Planeación, Unidad de Análisis Macroeconómico.

Pollack, M. y A. Uthoff (1990): Pobreza y empleo: un análisis del período 1969-1987 en el Gran Santiago, Documento de trabajo, $\mathrm{N}^{\circ} 348$, Santiago de Chile, Programa Regional del Empleo para América Latina y el Caribe (PREALC/OIT).

Psacharopoulos, G., S. Morley, A. Fiszbein, H. Lee, y B. Wood (1992): Poverty and income distribution in Latin America: The story of the 1980s, Report, $\mathrm{N}^{\circ} 27$, Washington, D.C., Banco Mundial.

Schulthess, W. y G. Demarco (1993): Argentina: evolución del sistema nacional de previsión social y propuesta de reforma, Santiago de Chile, CEPAL/PNUD.

Urani, A., A. Moreira y L.D. Willcox (1997): Choques, respailas de política económica e distribução de renda no Brasil, serie Financiamiento del desarrollo, $\mathrm{N}^{\mathrm{c}}$ 43, Santiago de Chile, CEPAL, Santiago, de Chile.

Uthoff, A. (1995): Reforma a los sistemas de pensiones en América Latina y el Caribe, serie Financiamiento del desarrollo, $\mathrm{N}^{\circ} 29$, Santiago de Chile, CEPAL.

Uthoff, A. y R. Szalachman (1991): Sistema de pensiones en América Latina. Diagnóstico y alternativas de reforma: Costa Rica, Ecuador, Uruguay y Venezuela, vol. 1, Santiago de Chile, CEPAL/PNUD.

(1992): Sistema de pensiones en América Latina, Diagnóstico y alternativas de reforma: Bolivia, Brasil, Colombia, Guatemala, México, vol, 2, Santiago de Chile, CEPAL/PNUD

(1994): Sistema de pensiones en América Latina. Diagnóstico y alternativas de reforma: El Salvador, Honduras, Jamaica, Paraguay y Trinidad y Tobago, vol. 3, Santiago de Chile, CEPAL/PNUD. 